\title{
Excited state spectroscopy of para di-substituted benzenes in a supersonic beam using resonant two photon ionization
}

\author{
R. Tembreull,* T. M. DunN and D. M. Lubman \\ Department of Chemistry, University of Michigan, Ann Arbor, Michigan 48109, U.S.A.
}

(Received 3 December 1985; accepted 31 January 1986)

\begin{abstract}
Excited state vibronic spectra of $p$-aminophenol, $p$-cresol, $p$-fluoroaniline, $p$-fluorophenol, hydroquinone and $p$-toluidine have been obtained using resonant two photon ionization supersonic beam mass spectrometry. Despite marked similarities in the spectra, notable differences exist and different para polyatomic substituents in the same molecule show vibronic evidence of their real molecular symmetry of $C_{2 v}$. Expansion of the ring is also noted upon excitation in all cases. Further, it is now evident that the assignment of some vibronic bands historically interpreted as sequence structure must be reconsidered since molecules like hydroquinone are mixtures of cis and trans and others have a vibronic structure arising from the polyatomic nature of the substituents $\left(\mathrm{cf} . \mathrm{CH}_{3}\right)$.
\end{abstract}

\section{INTRODUCTION}

Supersonic molecular beam techniques provide a powerful means of probing the excited state of polyatomic molecules [1-8]. Room temperature u.v.visible absorption spectra of aromatic hydrocarbons are typically broad and relatively featureless in contour. In contrast, absorption spectra in a supersonic jet characteristically are sharp $[9,10]$. This is due to the very intense cooling which occurs in the supersonic flow [11-14]. Two body collisions which convert thermal energy of random motion into kinetic energy of directed mass flow serve as the mechanism by which this cooling proceeds. As a result of such collisions the translational temperature of the expanding gas drops precipitously. Moreover, a small population of polyatomic molecules seeded into an expansion of an inert carrier gas undergoes relaxation into low energy rotational and vibrational levels. The rotational degrees of freedom of such molecules are nearly in equilibrium with the rate of translational cooling and will therefore cool to within a few degrees of absolute zero. Vibrational relaxation, on the other hand, is quite mode-dependent and much less efficient but it is nonetheless remarkable.

Resonant two photon ionization (R2PI) is a very useful spectroscopic tool at megawatt laser energies where, at least for relatively rigid molecules, the nonresonant background is small compared to the resonant intensity $[15,29]$. It is therefore possible to exploit the fact that absorption of the first photon occurs in resonance with a vibronic band. Absorption of the second photon pumps the molecule into the ionization continuum or a Rydberg state from which ionization can be made to occur. The cross section for the absorption of this second photon is not a strong function of wavelength and at least it appears to be devoid of sharp features for the polyatomic molecules of interest here [16]. Consequently, R2PI spectra of polyatomic molecules are, in effect, absorption spectra, although the total ion production is being monitored. R2PI can therefore be used as an excited state probe.
Several outstanding advantages of supersonic beam R2PI mass spectrometry are worthy of mention. Perhaps foremost is the ability of the supersonic beam to produce a nearly collisionless beam of monoenergetic gas phase molecules, thereby circumventing matrix effects which are present in solid state absorption spectra obtained under cryogenic conditions. Further, ionization can be made to occur in molecules which have a negligible fluorescence quantum yield. Thus, nonfluorescent molecules may be studied. It is also of significance that ion production and detection is much more efficient $[17,20-23]$ than molecular excitation and detection employed in conventional absorption spectroscopy so that one can probe the relatively low density of seed molecules in a supersonic expansion. In addition, this technique has produced soft ionization or only molecular ions with high efficiency $[18,24-29]$ at modest laser intensities for aromatic systems of interest studied thus far. Therefore, the molecular ions produced can be selectively monitored in a mass spectrometer so that there is no question as to the identity of the species studied versus possible background contamination due to formation of clusters.

\section{EXPERIMENTAL}

The experimental apparatus used in these experiments has been described elsewhere [30]. The device is constructed such that laser radiation is perpendicular to the supersonic beam. The interaction region is at a fixed distance of $9.25 \mathrm{~cm}$ from the nozzle thereby ensuring molecular "free flow". Orthogonal to both the jet and the laser beam a time of flight mass spectrometer based on the design of WILEY and MCLAREN [31] serves as the detection systcm. A dual microchannel plate (R. M. Jordan Co.) is used to monitor the signal.

A Quanta Ray PSV-1 pulsed nozzle provides the beam source in our experiments. The maximum rate of flow through the orifice, i.e. "choked flow", is reached when the fwhm of the gas pulse is $55 \mu \mathrm{s}$, so that maximum theoretical cooling should be achieved. Sample introduction is accomplished by flowing a steady stream of Ar carrier gas over a reservoir containing the solid or liquid. In some cases the reservoir was heated mildly to provide sufficient vapor pressure. Typical concentrations of seed molecules in the 
expansion are between 5 and 50 p.p.m. as determined by weight loss in the sample reservoir over an extended period of time. The background pressure in the reservoir is $1 \mathrm{~atm}$. An average chamber pressure of $1-2 \times 10^{-5}$ torr during pulsed valve operation is maintained with a $6^{\prime \prime}$ diffusion pump and a liquid $\mathrm{N}_{2}$ cooled baffle.

The laser source consists of the output of a Quanta Ray PDL-1A Nd:YAG laser. Tunable u.v. radiation is generated by frequency doubling the output of the dye in a phase matched $\mathrm{KD}^{*} \mathrm{P}$ crystal. This is performed using the Quanta Ray WEX-1 wavelength extension device. The near u.v. radiation which has an energy of $\sim 0.1-0.3 \mathrm{~mJ}$ at $10 \mathrm{~Hz}$ is then collimated with a telescope (positive lens, $30 \mathrm{~cm}$ focal length; negative lens $10 \mathrm{~cm}$ focal length) to $\mathrm{a} \sim 2 \mathrm{~mm}$ beam. A Quanta Ray CDM-1 Control Display Module is used to control the stepping motor which tunes the grating in the dye cavity.

Experiments were performed at the $10 \mathrm{~Hz}$ repetition rate of the laser system. The flight time of the molecular beam, which is on the order of $230 \mu \mathrm{s}$, requires proper synchronization between the pulsed valve and laser. The oscillator output from the Nd:YAG laser triggers the pulsed nozzle control unit which generates a high current pulse that causes the valve to open. An adjustable delay between the oscillator pulse and the pulse which triggers the nozzle provides a means of synchronizing the arrival of the molecular beam into the interaction region with the laser pulse. The laser then fires and triggers the oscilloscope and gated integrator. A Stanford Research System SRS 250 gated integrator unit was used to monitor the molecular ion peak in the TOFMS as a function of wavelength and the signal was displayed on a stripchart recorder. No correction was made for dye laser intensity in the spectra so obtained.

\section{RESULTS AND DISCUSSION}

Ionization spectra of the para substituted benzene derivatives $p$-aminophenol, $p$-cresol, $p$-fluoroaniline, $p$ fluorophenol, hydroquinone and toluidine are presented in Figs 1-6. We chose to investigate these particular compounds because of the importance of aromatic molecules in biological systems. Further, for para di-substituted benzenes which contain substituent groups of relatively light mass there is little mixing of the normal modes as described for benzene. Thus, the normal modes of such molecules resemble those of benzene and its para substituted analog $p$ difluorobenzene, both of which have well characterized vibrational modes. This is a crucial point since nomenclature which is in use for benzene may therefore be applied to the derivatives treated here. It must be clearly understood, however, that every molecule has its own unique normal modes of vibration. For example, the lowest frequency mode of p-diiodobenzene which is totally symmetric involves stretching of the iodine substituents and does not resemble the characteristic lowest ring mode of the lightly substituted benzenes. Thus, the over simplified treatment presented herein must be viewed with caution. Our intent in this work is to draw attention to the fact that these molecules, about whose $S_{1}$ state little is known,

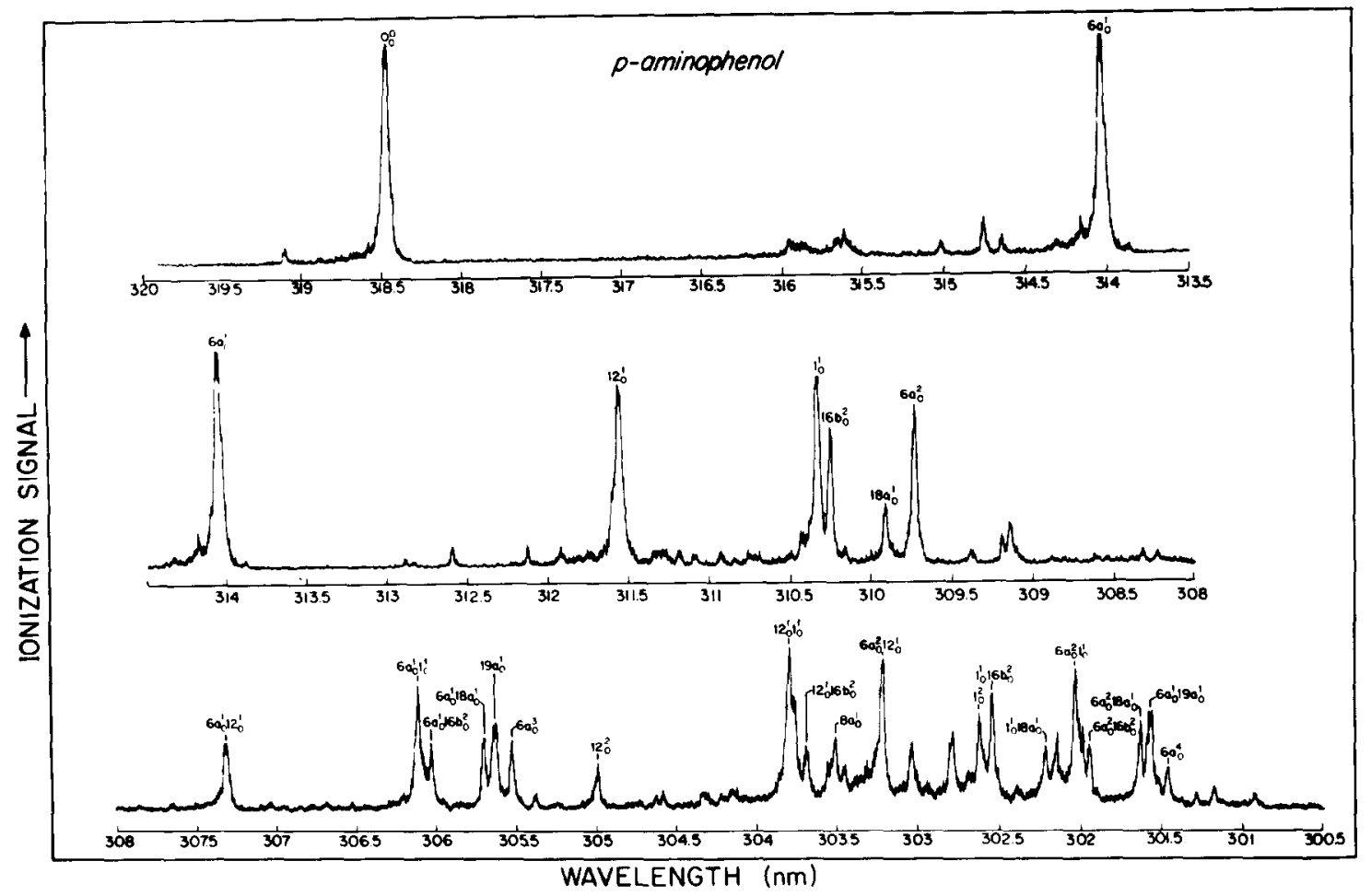

Fig. 1. Resonant two photon ionization spectra of p-aminophenol in a supersonic expansion of argon from 1 atm. backpressure. 


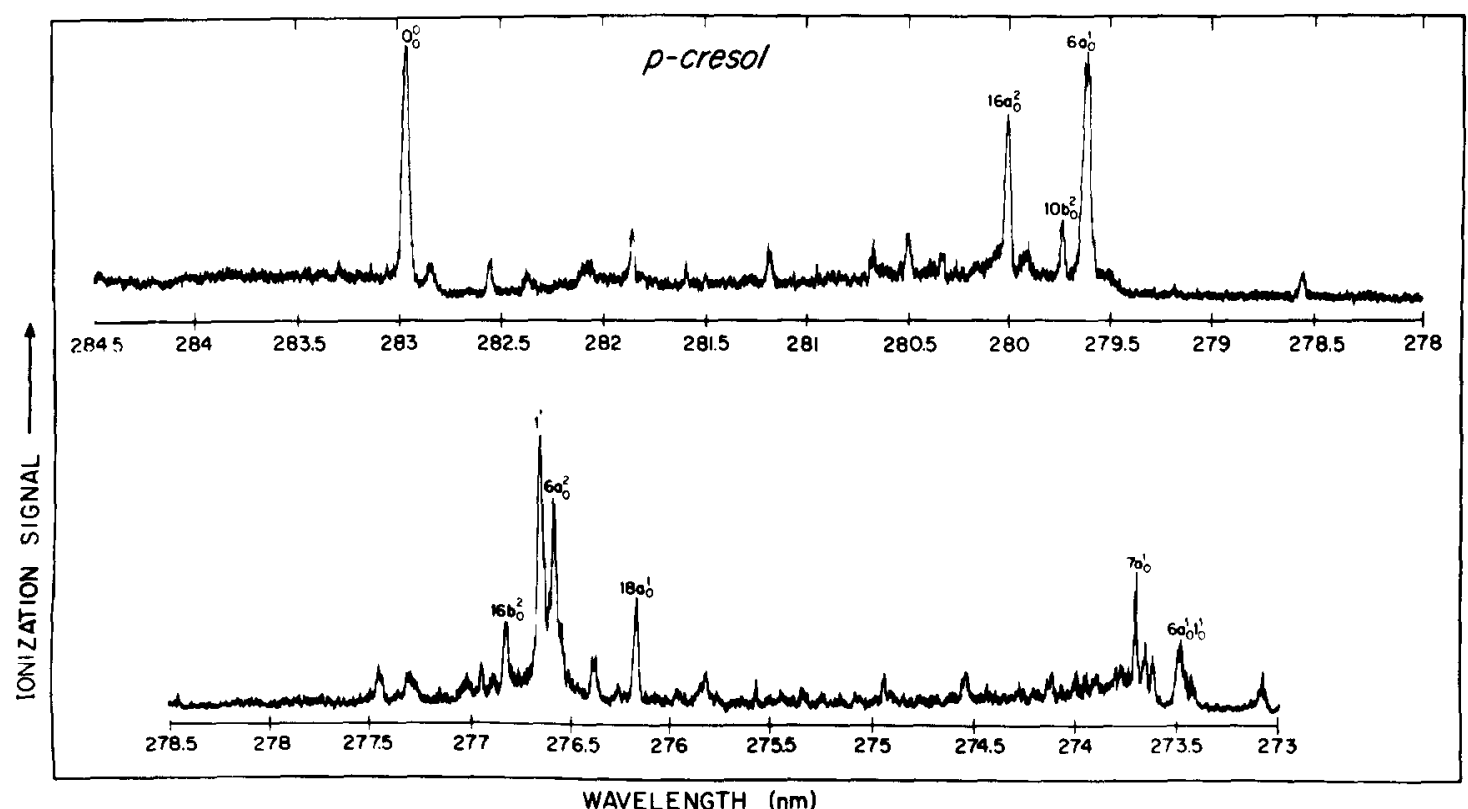

Fig. 2. Resonant two photon ionization spectra of $p$-cresol in a supersonic expansion of argon from 1 atm. backpressure.

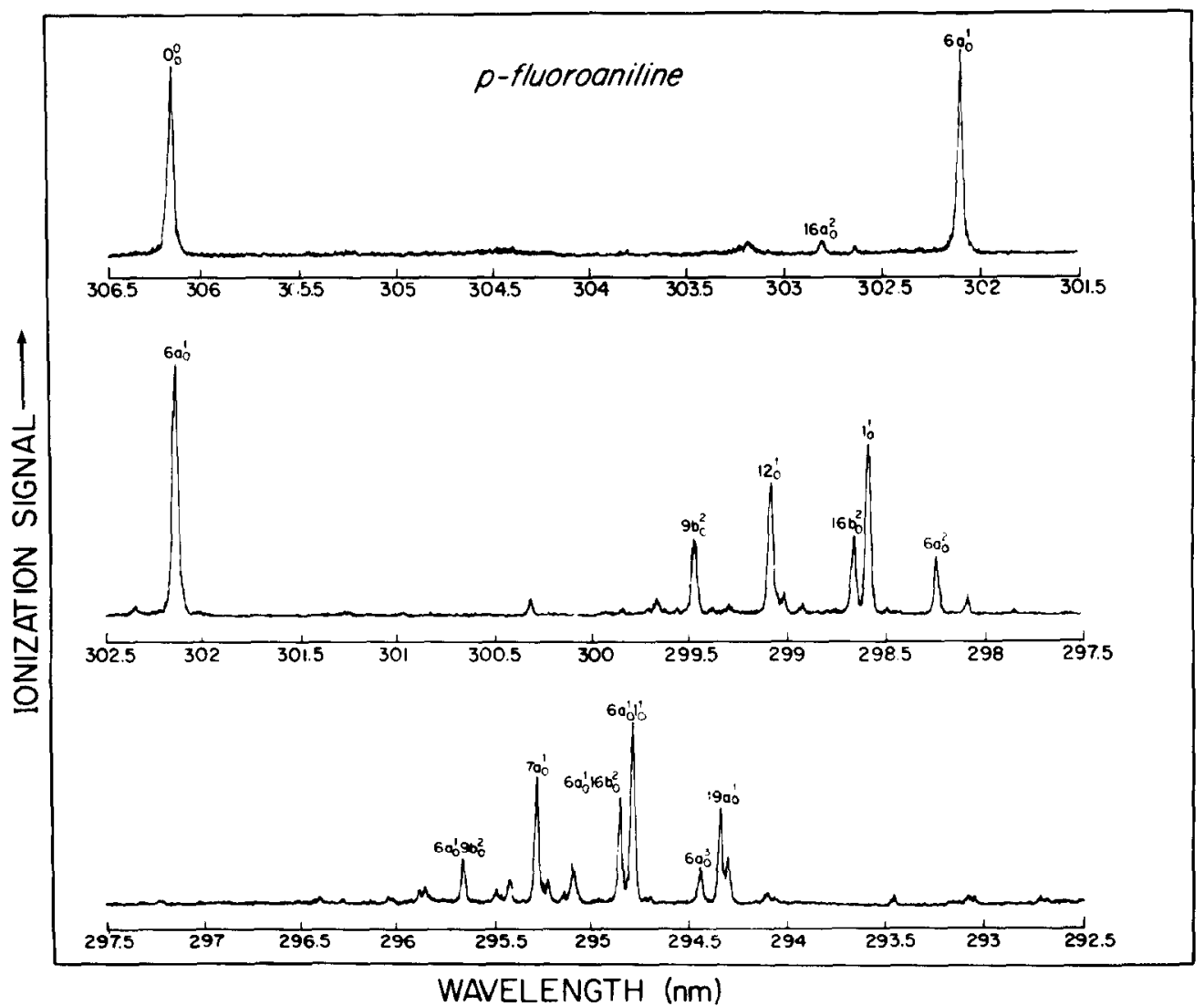

Fig. 3. Resonant two photon ionization spectra of p-fluoroaniline in a supersonic expansion of argon from $1 \mathrm{~atm}$. backpressure. 


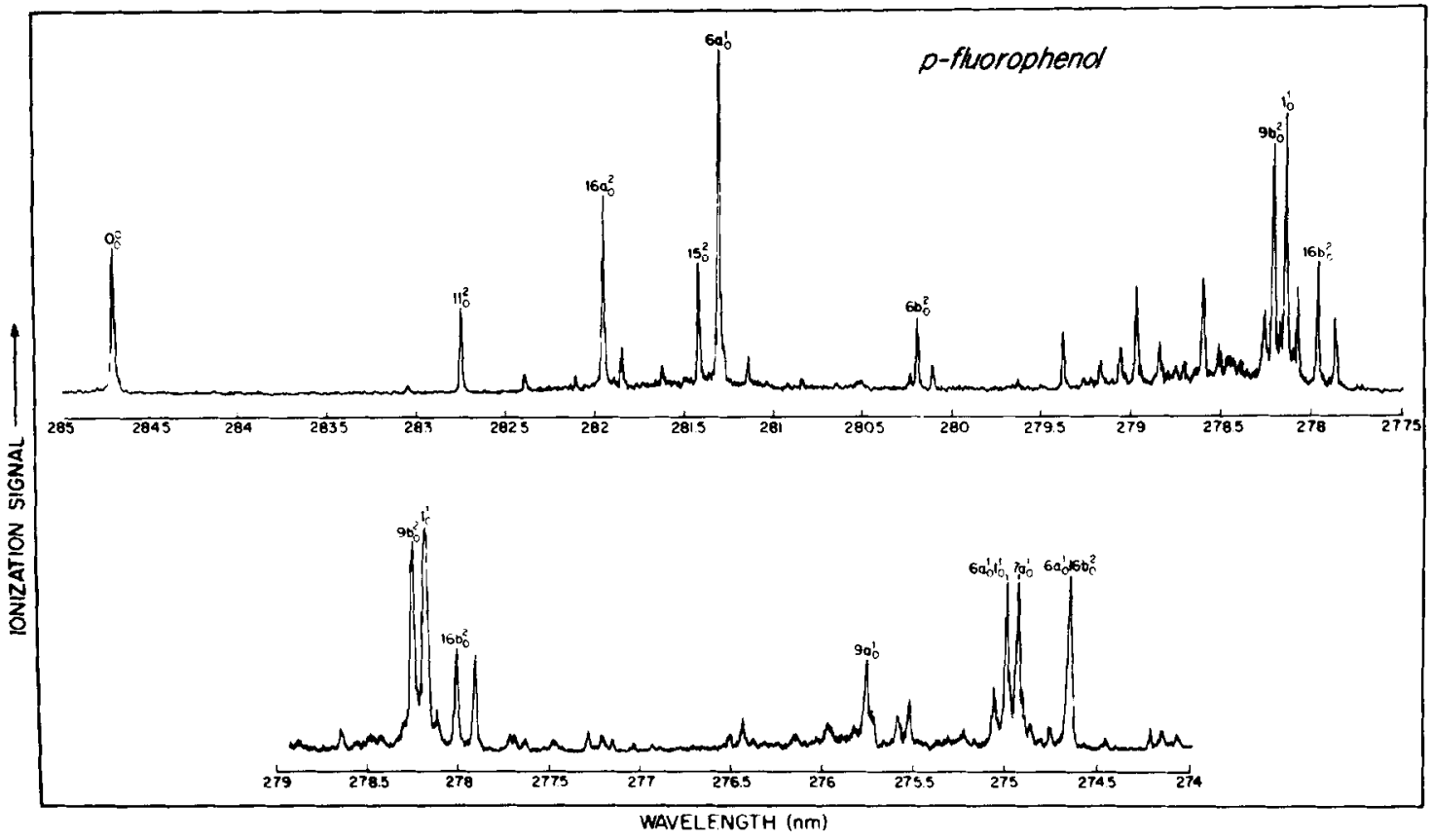

Fig. 4. Resonant two photon ionization spectra of $p$-fluorophenol in a supersonic expansion of argon from $1 \mathrm{~atm}$. backpressure.

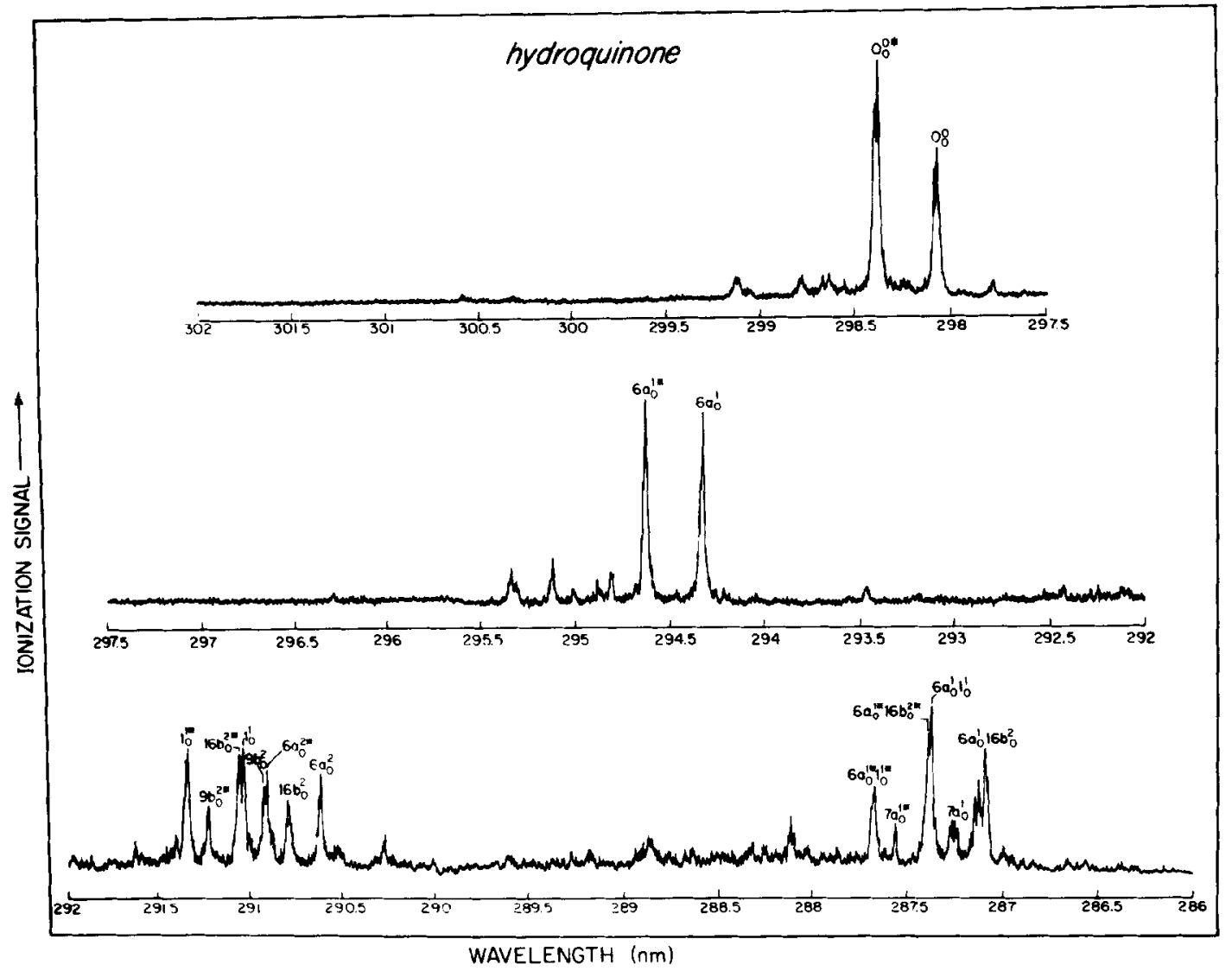

Fig. 5. Resonant two photon ionization spectra of hydroquinone in a supersonic expansion of argon from 1 atm. backpressure. 


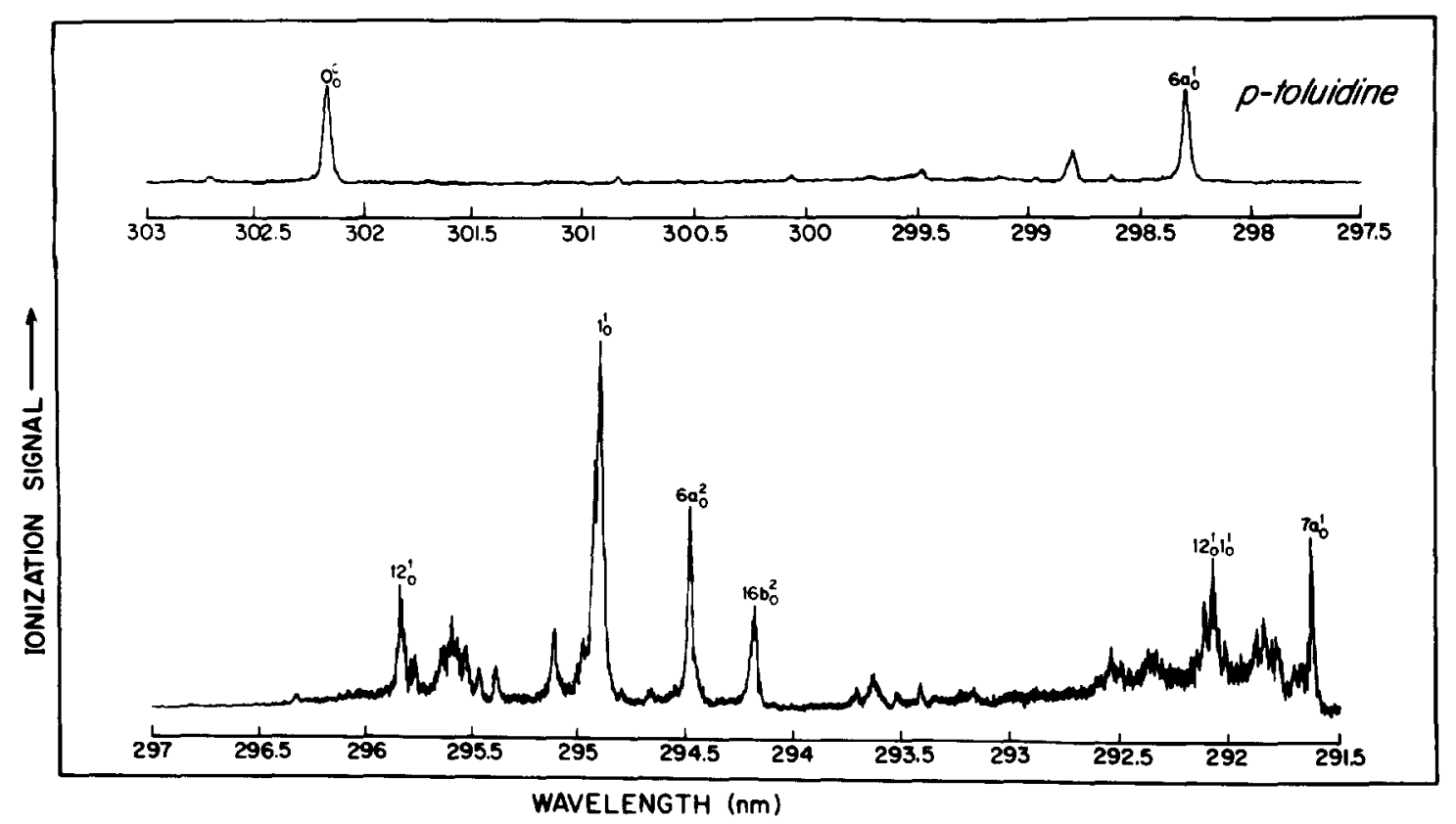

Fig. 6. Resonant two photon ionization spectra of $p$-toluidine in a supersonic expansion of argon from $1 \mathrm{~atm}$. backpressure.

have vibronic spectra which bear a close resemblance to one another but that, in the absence of "hot" vibrational bands and extended rotational contours, significant differences can also be seen.

Among the most intense bands in each spectrum are the vibronic transition to the totally asymmetric component of the doubly degenerate $\left(608 \mathrm{~cm}^{-1}\right) e_{2 g}$ mode $\left(v_{6 a}\right)$ of benzene which typically lies between $\sim 420-440 \mathrm{~cm}^{-1}$ and the aromatic "ring breathing" between $\sim 805-840 \mathrm{~cm}^{-1}\left(v_{1}\right)$. The appearance of other vibronic transitions with an intensity comparable to that of the $6 a_{0}^{1}$ and $1_{0}^{1}$ modes suggest that they also be interpreted as totally. symmetric. The number of these spectral features indicate that for the molecules under study the molecular symmetry are all $C_{2 v}$ in practice. Thus, para polyatomic substituents effectively determine the overall molecular symmetry by virtue of their local geometry. In $p$-cresol, for example, the band at $871 \mathrm{~cm}^{-1}$, which is too intense to be an overtone, is assigned as $18 a_{0}^{1}$. Other active totally symmetric modes we report in this study include transitions designated $8 a, 7 a, 19 a$ and 12 [32,33]. Absorption of a photon sufficient to excite overtones of ring bending modes in the $690-850 \mathrm{~cm}^{-1}$ region also accounts for bands of considerable intensity. The most active of these modes is believed to be the out-ofplane bending mode $16 b$ and the in-plane bending mode $9 b$. Table 1 summarizes the assignment of the major bands.

Conducting the experiment in a supersonic expansion of Ar results in a significant cooling of the vibrational degrees of freedom. As a result virtually all vibronic transitions originate from the $v^{\prime \prime}=0$ level of the ground state. The lack of sequence structure which accompanies the cooling process therefore simplifies the spectra significantly and relatively few vibronic bands are observed. Thus, totally symmetric bands are conspicuous because of their intensity, whereas low

Table 1.

\begin{tabular}{|c|c|c|c|c|c|c|c|}
\hline Mode & $\begin{array}{c}\text { Type of } \\
\text { vibration }\end{array}$ & p-Aminophenol & p-Cresol & p-Fluoroaniline & $p$-Fluorophenol & Hydroquinone $†$ & p-Toluidine \\
\hline $6 a_{0}^{1}$ & IP $\mathrm{CCC} \sigma$ & 443 & 421 & 435 & 424 & $434^{*}, 435$ & 434 \\
\hline $12_{0}^{1}$ & IP C-Hv & 698 & & 775 & & & 707 \\
\hline $9 b_{0}^{2}$ & IP $\sigma$ & & & 731 & 817 & & \\
\hline $16 b_{0}^{2}$ & OOPa & 833 & 786 & 821 & & $853^{*}, 850$ & 898 \\
\hline 11 & Ring breathing & 823 & 808 & 830 & 825 & $820^{*}, 819$ & 815 \\
\hline $18 a_{0}^{1}$ & IP C-H $v$ & 868 & 871 & & & & \\
\hline $7 a_{0}^{1}$ & $\mathrm{C}-\mathrm{X} \boldsymbol{y}$ & & 1199 & 1206 & 1245 & $1267 *, 1268$ & 1194 \\
\hline $19 a_{0}^{1}$ & IPv & 1318 & & 1315 & & & \\
\hline $8 a_{0}^{1}$ & IP C-H $v$ & 1549 & & & & & \\
\hline
\end{tabular}

†The asterisk is used to denote a distinct structural isomer [38]. 
frequency bands which are less intense can be correlated with two quanta of nontotally symmetric modes. This is a major advantage over the relatively high temperature absorption spectra in the existing literature. It is now evident, for example, that some bands which were historically interpreted as sequence structure were incorrectly assigned. For instance, because of the absence of a hot band $38 \mathrm{~cm}^{-1}$ from the electronic origin of $p$-cresol in the jet spectrum, it is plausible to regard many of the low frequency modes of the $p$-cresol molecule as torsional vibrations of the methyl group rather than sequences [34].

Slight expansion of the aromatic ring is a general property of these molecules upon electronic excitation. This is borne out by the appearance of totally symmetric fundamentals characteristic of the benzene ring which appear consistently throughout the spectra, in particular, $6 a_{0}^{1}$ and $1_{0}^{1}$. Ring expansion is indicated by the appearance of these fundamentals at slightly lower frequencies to those of the corresponding ground state frequencies. Severe geometric deformation such as an excited state equilibrium geometry in which the ring is puckered would lead to drastic changes in the number, type and frequency of active vibronic bands in the spectra and have not been observed.

Unequivocal assignment of vibronic bands is difficult due to the lack of complementary data. Infrared and Raman spectra of the excited state would provide a wealth of information about the symmetry of the active transitions. In conjunction with fluorescence spectra such data would make it possible to definitively assign specific transitions through the use of combination rulcs. To date, however, these conventional spectroscopic techniques have been plagued with difficulties. For example, all of the compounds investigated here have very low fluorescence quantum yields. Despite the lack of excited state spectral information available from other spectroscopic techniques reasonable assignments were made possible from ground state spectra $[33,35]$ on the basis of the criteria cited below. These criteria are based on principles discussed above [36, 37].

1. Symmetry was considered to be $C_{20}$.

2. For para substituted derivatives with small substituents severe shape deformation between the ground and excited state equilibrium geometries was deemed unlikely.

3. Vibronic bands of the largest intensity are totally symmetric and should appear in progressions, e.g. $6 a_{0}^{1}, 6 a_{0}^{2}, 6 a_{0}^{3}, \ldots$

4. Nontotally symmetric modes absorb an even number of vibrational photon quanta and will rarely absorb more than two, i.e. $18 a_{0}^{2},\left(18 a_{0}^{4}\right)$ etc.

5. Hot bands which are not cooled are likely to be $1 \rightarrow 1$ sequence bands of low frequency bending vibrations. Such bands often appear to the red of the electronic origin and in combination with other modes.

The analyses presented in Tables 2-7 were made with the aid of these guidelines. It is essential to point
Table 2. p-Aminophenol

\begin{tabular}{|c|c|c|c|}
\hline$\underset{(\mathrm{nm})}{\lambda}$ & $\left(\begin{array}{c}v \\
\left(\mathrm{~cm}^{-1}\right)\end{array}\right.$ & $\begin{array}{c}\Delta v \\
\left(\mathrm{~cm}^{-1}\right)\end{array}$ & Assignment \\
\hline 318.48 & 31390 & 0 & $0_{0}^{0}$ \\
\hline 314.05 & 31833 & 443 & $6 a_{0}^{1}$ \\
\hline 311.55 & 32088 & 698 & $12_{0}^{1}$ \\
\hline 310.34 & 32213 & 823 & $1{ }_{0}^{1}$ \\
\hline 310.25 & 32223 & 833 & $16 b_{0}^{2}$ \\
\hline 309.91 & 32258 & 868 & $18 a_{0}^{1}$ \\
\hline 309.73 & 32277 & 887 & $6 a_{0}^{2}$ \\
\hline 309.15 & 32337 & 947 & $\gamma \mathbf{N H}_{20}^{2}$ \\
\hline 307.34 & 32528 & 1138 & $6 a_{0}^{1} 12_{0}^{1}$ \\
\hline 306.14 & 32655 & 1265 & $6 a_{0}^{1} 1_{0}^{1}$ \\
\hline 306.05 & 32665 & 1275 & $6 a_{0}^{1} 16 b_{0}^{2}$ \\
\hline 305.73 & 32699 & 1309 & $6 a_{0}^{1} 18 a_{0}^{1}$ \\
\hline 305.65 & 32708 & 1318 & $19 a_{0}^{1}$ \\
\hline 305.56 & 32717 & 1327 & $6 a_{0}^{3}$ \\
\hline 305.00 & 32777 & 1387 & $12_{0}^{2}$ \\
\hline 303.79 & 32908 & 1518 & $12_{0}^{1} 1_{0}^{1}$ \\
\hline 303.68 & 32920 & 1530 & $12_{0}^{1} 16 b_{0}^{2}$ \\
\hline 303.50 & 32939 & 1549 & $8 a_{0}^{1}$ \\
\hline 303.21 & 32971 & 1581 & $6 a_{0}^{2} 12_{0}^{1}$ \\
\hline 303.05 & 32988 & 1598 & \\
\hline 302.79 & 33017 & 1627 & \\
\hline 302.63 & 33034 & 1644 & $1_{0}^{2}$ \\
\hline 302.55 & 33043 & 1653 & $1_{0}^{1} 16 b_{0}^{2}$ \\
\hline 302.21 & 33080 & 1690 & $1_{0}^{1} 18 a_{0}^{1}$ \\
\hline 302.15 & 33087 & 1697 & \\
\hline 302.04 & 33099 & 1709 & $6 a_{0}^{2} 1_{0}^{1}$ \\
\hline 301.95 & 33108 & 1718 & $6 a_{0}^{2} 16 b_{0}^{2}$ \\
\hline 301.64 & 33142 & 1752 & $6 a_{0}^{2} 18 a_{0}^{1}$ \\
\hline 301.58 & 33149 & 1759 & $6 a_{0}^{1} 19 a_{0}^{1}$ \\
\hline 301.46 & 33162 & 1772 & $6 a_{0}^{4}$ \\
\hline
\end{tabular}

Table 3. p-Cresol

\begin{tabular}{cccl}
\hline $\begin{array}{c}\lambda \\
(\mathrm{nm})\end{array}$ & $\begin{array}{c}v \\
\left(\mathrm{~cm}^{-1}\right)\end{array}$ & $\begin{array}{c}\Delta v \\
\left(\mathrm{~cm}^{-1}\right)\end{array}$ & \multicolumn{1}{c}{ Assignment } \\
\hline 282.97 & 35329 & 0 & $0_{0}^{0}$ \\
280.02 & 35701 & 372 & $16 a_{0}^{2}$ \\
279.75 & 35736 & 407 & $10 b_{0}^{2}$ \\
279.64 & 35750 & 421 & $6 a_{0}^{1}$ \\
276.82 & 36115 & 786 & $16 b_{0}^{2}$ \\
276.64 & 36137 & 808 & $11_{0}^{1}$ \\
276.57 & 36148 & 819 & $6 a_{0}^{2}$ \\
276.16 & 36200 & 871 & $18 a_{0}^{1}$ \\
273.68 & 36528 & 1199 & $7 a_{0}^{1}$ \\
273.45 & 36559 & 1230 & $6 a_{0}^{1} 1_{0}^{1}$ \\
\hline
\end{tabular}

out that the experimental spectra were correlated with observed room temperature ground state i.r. and Raman spectra in the gas and/or liquid phases. Thus, it must be emphasized that the interpretations presented here can only be considered reasonable. Whereas it is generally easy to interpret totally symmetric bands which occur in progressions, fundamentals which are relatively weak in intensity and those bands attributed to two quanta of nontotally symmetric modes may be subject to alternative interpretations. Further, heating the sample prior to expansion in the supersonic jet is an inadequate means of identifying hot bands by changes in signal intensity. This is largely due to the fact that the statistical distribution of populated vibrational states 
Table 4. $p$-Fluoraniline

\begin{tabular}{|c|c|c|c|}
\hline $\begin{array}{c}\lambda \\
(\mathrm{nm})\end{array}$ & $\left(\begin{array}{c}v \\
\left(\mathrm{~cm}^{-1}\right)\end{array}\right.$ & $\begin{array}{c}\Delta v \\
\left(\mathrm{~cm}^{-1}\right)\end{array}$ & Assignment \\
\hline 306.20 & 32649 & 0 & $0_{0}^{9}$ \\
\hline 302.80 & 33015 & 366 & $16 a_{0}^{2}$ \\
\hline 302.17 & 33084 & 435 & $6 a_{0}^{1}$ \\
\hline 299.49 & 33380 & 731 & $9 b_{0}^{2}$ \\
\hline 299.10 & 33424 & 775 & $12_{0}^{1}$ \\
\hline 298.69 & 33470 & 821 & $16 b_{0}^{2}$ \\
\hline 298.61 & 33479 & 830 & $1_{0}^{1}$ \\
\hline 298.26 & 33518 & 869 & $6 a_{0}^{2}$ \\
\hline 295.66 & 33813 & 1164 & $6 a_{0}^{1} 9 b_{0}^{2}$ \\
\hline 295.29 & 33855 & 1206 & $6 a_{0}^{1} 12_{0}^{1}$ \\
\hline 295.11 & 33876 & 1227 & $7 a_{0}^{1}$ \\
\hline 294.86 & 33904 & 1255 & $6 a_{0}^{1} 16 b_{0}^{2}$ \\
\hline 294.79 & 33913 & 1264 & $6 a_{0}^{1} 1_{0}^{1}$ \\
\hline 294.45 & 33952 & 1303 & $6 a_{0}^{3}$ \\
\hline 294.34 & 33964 & 1315 & $19 a_{0}^{1}$ \\
\hline 292.29 & 34203 & 1554 & $12_{0}^{2}$ \\
\hline 292.24 & 34208 & 1559 & $9 b_{0}^{2} 1_{0}^{1}$ \\
\hline 291.95 & 34242 & 1593 & $12_{0}^{1} 16 b_{0}^{2}$ \\
\hline 291.90 & 34248 & 1599 & $12_{0}^{1} 1_{0}^{1}$ \\
\hline 291.75 & 34266 & 1617 & \\
\hline 291.57 & 34287 & 1638 & $6 a_{0}^{2} 12_{0}^{1}$ \\
\hline 291.46 & 34300 & 1651 & $16 b_{0}^{2}$ \\
\hline 291.40 & 34307 & 1658 & $1_{0}^{2}$ \\
\hline 291.13 & 34339 & 1690 & $6 a_{0}^{2} 16 b_{0}^{2}$ \\
\hline 291.07 & 34346 & 1697 & $6 a_{0}^{2} 1_{0}^{1}$ \\
\hline 290.63 & 34398 & 1749 & $6 a_{0}^{1} 19 a_{0}^{1}$ \\
\hline 288.25 & 34682 & 2033 & $6 a_{0}^{3} 9 b_{0}^{2}$ \\
\hline 287.80 & 34736 & 2087 & $6 a_{0}^{1} 16 b_{0}^{2} 1_{0}^{1}$ \\
\hline 287.34 & 34792 & 2143 & $1_{0}^{1} 19 a_{0}^{1}$ \\
\hline
\end{tabular}

Table 5. p-Fluorophenol

\begin{tabular}{cccl}
\hline $\begin{array}{c}\lambda \\
(\mathrm{nm})\end{array}$ & $\begin{array}{c}\nu \\
\left(\mathrm{cm}^{-1}\right)\end{array}$ & $\begin{array}{c}\Delta v \\
\left(\mathrm{~cm}^{-1}\right)\end{array}$ & \multicolumn{1}{c}{ Assignment } \\
\hline 284.69 & 35116 & 0 & $0_{0}^{0}$ \\
282.75 & 35357 & 241 & $11_{0}^{2}$ \\
281.94 & 35458 & 342 & $16 a_{0}^{2}$ \\
281.41 & 35525 & 409 & $15_{0}^{2}$ \\
281.29 & 35540 & 424 & $6 a_{0}^{1}$ \\
280.18 & 35681 & 565 & $6 b_{0}^{1}\left(\right.$ or $\left.10 b_{0}^{2}\right)$ \\
278.97 & 35836 & 720 & $\tau \mathrm{OH}_{0}^{2}$ \\
278.61 & 35882 & 766 & $16 b_{0}^{2}$ \\
278.21 & 35933 & 817 & $9 b_{0}^{2}$ \\
278.15 & 35941 & 825 & $1_{0}^{1}$ \\
275.76 & 36253 & 1137 & $9 a_{0}^{1}$ \\
275.00 & 36353 & 1237 & $6 a_{0}^{1} 1_{0}^{1}$ \\
274.94 & 36361 & 1245 & $7 a_{0}^{1}$ \\
274.66 & 36398 & 1282 & $6 a_{0}^{1} 16 b_{0}^{2}$ \\
\hline
\end{tabular}

is little affected by these small temperature changes. It is also clear that the resonant signal intensity in R2PI is very sensitive to experimental conditions since the signal is proportional to the square of the incident laser intensity. Estimates of relative peak heights are thus no better than $\sim 25 \%$.

In conclusion ionization spectra obtained in the present work indicate a striking similarity between the normal vibrational modes of "lightly" substituted benzenes in the excited state. With the aid of such spectral data significant progress can be made toward a more complete assignment and characterization of the
Table 6. Hydroquinone†

\begin{tabular}{cccl}
\hline $\begin{array}{c}\lambda \\
(\mathrm{nm})\end{array}$ & $\begin{array}{c}v \\
\left(\mathrm{~cm}^{-1}\right)\end{array}$ & \multicolumn{1}{c}{$\begin{array}{c}\Delta v \\
\left(\mathrm{~cm}^{-1}\right)\end{array}$} & \multicolumn{1}{c}{ Assignment } \\
\hline 298.43 & 33499 & $0^{*}$ & $0_{0}^{0 *}$ \\
298.13 & 33533 & 0 & $0_{0}^{0}$ \\
294.61 & 33933 & $434^{*}$ & $6 a_{0}^{1 *}$ \\
294.31 & 33968 & 435 & $6 a_{0}^{1}$ \\
291.30 & 34319 & $820^{*}$ & $1_{0}^{1 *}$ \\
291.20 & 34331 & $832^{*}$ & $9 b_{0}^{2 *}$ \\
291.02 & 34352 & $853^{*}, 819$ & $16 b_{0}^{2 *}, 1_{0}^{1}$ \\
290.89 & 34367 & $868^{*}, 834$ & $6 a_{0}^{2 *}, 9 b_{0}^{2}$ \\
290.76 & 34383 & 850 & $16 b_{0}^{2}$ \\
290.60 & 34401 & 869 & $6 a_{0}^{2}$ \\
287.66 & 34753 & $1254^{*}$ & $6 a_{0}^{1 *}, 1_{0}^{1 *}$ \\
287.55 & 34766 & $1267^{*}$ & $7 a_{0}^{1 *}$ \\
287.38 & 34787 & $1288^{*}, 1254$ & $6 a_{0}^{1 *} 16 b_{0}^{2 *}, 6 a_{0}^{1} 1_{0}^{1}$ \\
287.26 & 34801 & 1268 & $7 a_{0}^{1}$ \\
287.10 & 34821 & 1288 & $6 a_{0}^{1} 16 b_{0}^{2}$ \\
\hline
\end{tabular}
[38]

† The asterisk is used to denote a distinct structural isomer

Table 7. p-Toluidine

\begin{tabular}{cccl}
\hline $\begin{array}{c}\lambda \\
(\mathrm{nm})\end{array}$ & $\begin{array}{c}v \\
\left(\mathrm{~cm}^{-1}\right)\end{array}$ & $\begin{array}{c}\Delta v \\
\left(\mathrm{~cm}^{-1}\right)\end{array}$ & Assignment \\
\hline 302.16 & 33085 & 0 & $0_{0}^{0}$ \\
298.25 & 33519 & 434 & $6 a_{0}^{1}$ \\
295.84 & 33792 & 707 & $12_{0}^{1}$ \\
294.90 & 33900 & 815 & $1_{0}^{1}$ \\
294.48 & 33948 & 863 & $6 a_{0}^{2}$ \\
294.18 & 33983 & 898 & $16 b_{0}^{2}$ \\
292.09 & 34226 & 1141 & $6 a_{0}^{1} 12_{0}^{1}$ \\
291.64 & 34279 & 1194 & $7 a_{0}^{1}$ \\
291.19 & 34332 & 1247 & $6 a_{0}^{1} 1_{0}^{1}$ \\
290.68 & 34392 & 1307 & $6 a_{0}^{3}$ \\
288.90 & 34603 & 1518 & $12_{0}^{1} 1_{0}^{1}$ \\
288.80 & 34616 & 1531 & \\
288.00 & 34712 & 1627 & $6 a_{0}^{1} 7 a_{0}^{1}$ \\
287.94 & 34719 & 1634 & $1_{0}^{2}$ \\
287.56 & 34765 & 1680 & $6 a_{0}^{2} 1_{0}^{1}$ \\
287.05 & 34827 & 1742 & $6 a_{0}^{4}$ \\
\hline
\end{tabular}

normal modes of these molecules. We have shown that in some cases, in particular p-cresol, that bands that were previously attributed to sequence structure must in fact have an alternative interpretation. We also expect that identification of the number and frequency of the fundamentals will facilitate a deeper understanding of the excited state molecular potential as well as a better comprehension of the interaction between vibrational modes in these cases. Further, excited state molecular geometry can be deduced from the spectra obtained. The results indicate an expansion of the aromatic ring which occurs with retention of molecular symmetry and implicate the polyatomic para substituents in the lowering of molecular point group from that of a model $D_{2 h}$ compound such as $p$ difluorobenzene.

Acknowledgements-We gratefully acknowledge financial support from a Cottrell Research Grant and the donors of the 
Petroleum Research Fund, administered by the American Chemical Society. We acknowledge support of this work under NSF Grant CHE 83-19383 and partial support from the Army Research Office under Grant DAAG 29-85-K-1005. We wish to thank CHUNG HaNG Sin and Ho Ming PANG for technical assistance.

\section{REFERENCES}

[1] D. H. Levy, L. Wharton, R. E. Smalley, Chemical and Biochemical Applications of Lasers, Vol. II, p. 1. Academic Press, New York (1977).

[2] R. E. Smalley, L. Wharton and D. H. Levy, J. chem. Phys. 63, 4977 (1975).

[3] A. Amirav, U. Even and J. Jortner, J. chem. Phys. 71, 2319 (1979).

[4] A. Amirav, U. Even and J. Jortner, J. Analyt. Chem. 54, 1666 (1982).

[5] A. Amirav, U. Even and J. Jortner, J. phys. Chem. 85, 309 (1981)

[6] T. G. DieTz, M. A. Duncan, M. G. Liverman and R. E. SMalley, Chem. Phys. Lett. 70, 246 (1980).

[7] T. G. Dietz, M. A. Duncan, M. G. Liverman and R. E. SMalley, Chem. Phys. Lett. 73, 4816 (1980).

[8] R. E. Smalley, L. Wharton and D. H. Levy, Acc. Chem. Res. 10, 139 (1977).

[9] C. H. Sin, R. Tembreull and D. M. Lubman, Analyt. Chem. 56, 2776 (1984).

[10] R. Tembreull and D. M. Lubman, Analyt. Chem. 56, 1962 (1984).

[11] A. KANTROWITZ and J. GREY, Rev. Sci. Instrum. 22, 328 (1951).

[12] J. B. Anderson, R. P. Andres and J. B. FenN, Adv. Chem. Phys. 10, 275 (1966).

[13] G. M. MCClelland, K. L. Saenger, J. J. Valentini and D. R. Herschbach, J. Phys. Chem. 83, 947 (1979).

[14] R. E. Smalley, L. Wharton and D. H. LeVy, Acc. Chem. Res. 10, 139 (1977).

[15] D. M. Lubman, C. T. RetTNeR and R. N. ZARE, J. phys. Chem. 86, 1129 (1982).

[16] C. T. RetTner and J. H. Brophy, Chem. Phys. 24, 89 (1981).
[17] D. M. Lubman, R. NaAman and R. N. Zare, J. chem. Phys. 72, 3034 (1980).

[18] T. G. DieTz, M. A. Duncan, M. G. Liverman and R. E Smalley, J. chem. Phys. 73, 4816 (1980).

[19] R. Frueholz, J. WesSel and E. Wheatley, Analyt. Chem. 52, 281 (1980).

[20] M. Seaver, J. W. Hudgens and J. J. DeCorpo, Int. J. Mass Spectrom. Ion Phys. 34n, 159 (1980).

[21] U. BoesL, H. J. Neusser and E. W. Schlag, Chem. Phys. 55, 193 (1981).

[22] C. Klimcak and J. Wessel, Analyt. Chem. 52, 1283 (1980).

[23] J. BROPHY and C. T. RETTNER, Opt. Lett. 4, 337 (1979).

[24] L. ZANDEE and R. B. BERNSTEIN, J.chem. Phys. 70, 2574 (1979).

[25] L. ZANDEE and R. B. Bernstein, J. chem. Phys. 71, 1359 (1979).

[26] D. A. Lichtin, S. Datta-Ghosh, K. R. Newton and R. B. Bernstein, Chem. Phys. Lett. 75, 214 (1980).

[27] U. Boesl, H. J. Neusser and E. W. SchlaG, J. chem. Phys. 72, 4327 (1980).

[28] C. D. Cooper, A. D. Williamson, J. C. Miller and R. N. Compton, J. chem. Phys. 73, 1527 (1980).

[29] V. S. ANTONOV, I. N. KNYAZEV, V. S. LETOKHOV, V. M. Matuik, V. G. Moshev and V. K. PotaPov, Opt. Lett. 3, 37 (1978).

[30] D. M. Lubman and M. N. Kronick, Analyt. Chem. 54, 660 (1982).

[31] W. C. Wiley, I. H. MCLaRen, Rev. Sci. Instr. 26, 1150 (1955).

[32] G. VARSANYI, Vibrational Spectra of Benzene Derivatives, p. 71. Academic Press, New York (1969).

[33] G. VARSANYI, Assignments for Vibrational Spectra of Benzene Derivatives. Wiley, New York (1974).

[34] P. R. RAO, Ind. Acad. Sci. 55, 232 (1962).

[35] A. StoJILJKovic and D. H. WhIFFEN, Spectrochim. Acta 12, 47 (1958).

[36] M. ORCHIN and H. H. JAFFE, Symmetry, Orbitals and Spectra. Wiley, New York (1971).

[37] H. Sponer and E. Teller, Rev. Mod. Phys. 13, 75 (1941).

[38] T. M. Dunn, R. Tembreull and D. M. Lubman, Chem. Phys. Lett. 121, 453 (1985). 